\title{
Radiographic study of Sever's disease
}

\author{
YAN GAO $^{1}$, JIA LIU ${ }^{2}$, YIFANG $\mathrm{LI}^{2}$, QING LIU ${ }^{2}$, and SHUHUA XU ${ }^{1}$ \\ ${ }^{1}$ Department of Radiology and ${ }^{2}$ MRI Room, People's Hospital of Rizhao, Rizhao, Shandong 276800, P.R. China
}

Received November 25, 2019; Accepted December 13, 2019

DOI: $10.3892 /$ etm. 2020.8796

\begin{abstract}
X-ray and MRI differences of calcaneal epiphysis between Sever's disease patients and normal children were compared and analyzed to raise awareness of Sever's disease. A total of 98 children with Sever's disease were enrolled in the study, including 72 males and 26 females. Among them, 6 patients underwent MRI. There were 120 patients in the control group, including 73 males and 47 females, aged 8 to 15 years. The calcaneus epiphysis density (compact and cancellous bone type), the difference of the radiolucent line and the signal changes of Sever's disease on MRI of the two groups were observed and analyzed. Among the patients with Sever's disease, the male incidence rate was $79.59 \%$, the female incidence rate was $20.41 \%$, the average age of onset was 11.35 , the average age of onset for male was 11.49 , and the average age of onset for female was 10.80 . There were significant statistical differences between the two groups in terms of epiphysis density (compact and cancellous type) and translucent line $\left(\chi^{2}=38.85,137.51, \mathrm{P}<0.05\right)$. Six cases of MRI showed partial or complete different degrees of bone marrow, ligament, soft tissue edema, and joint effusion. Sever's disease is mainly characterized by increased density of the calcaneus epiphysis and a radiolucent line of the epiphysis. It manifests as heel edema on MRI image.
\end{abstract}

\section{Introduction}

The calcaneus epiphysitis, also known as Sever's disease, was first reported in detail by James Warren Sever in 1912. The diseased population is child and adolescent who have emerged and have not closed the calcaneal epiphysis. The clinical manifestations are localized pain and swelling of the heel. The positive medial-lateral compression test is helpful for the diagnosis of the disease. Many factors such as chronic injury, acute strain and cold stimulation can cause pain in the calcaneal nodules, and high-intensity physical activity

Correspondence to: Dr Yifang Li, MRI Room, People's Hospital of Rizhao, 126 Tai'an Road, Rizhao, Shandong 276800, P.R. China E-mail: 17725b@163.com

Key words: Calcaneus epiphysitis, calcaneal epiphysis, radiography, magnetic resonance imaging can aggravate the pain (1). Its pathogenesis is diverse, and the basic pathology is that the calcaneal callus is subjected to repetitive microfracture damage, causing aseptic inflammation of the calcaneal epiphysis, i.e. surrounding soft tissue and ligament $(2,3)$. There is currently no single explanation for all cases of the disease. The X-ray shows an increase in the density of the calcaneal epiphysis, and a radiolucent line of the epiphysis - that is, a fragmentation of the epiphysis $(4,5)$, but this phenomenon can also occur in healthy children and adolescents (6). Because the mechanical structure of the calcaneus is relatively weak, it is prone to injury (7). This study compared the X-ray image of 98 cases of Sever's disease patients and 120 cases of healthy children and adolescents. Among the 98 patients, 6 underwent MRI, the images showed abnormal signal. Statistical analysis and images processing was performed to provide a basis for the diagnosis of Sever's disease.

\section{Patients and methods}

General information. Children aged 8-15 years (8-10) who underwent X-ray examination of the lateral ankle or lateral calcaneus in People's Hospital of Rizhao (Rizhao, China) from May 2014 to April 2018 were retrospectively collected. There were 98 patients diagnosed as Sever's disease, including 72 males and 26 females. A total of 120 healthy children and adolescents were also collected, 73 males and 47 females. Of the 98 patients, 6 underwent MRI. Inclusion criteria: Complete and accurate personal information, clear images, no metabolic and endocrine disorders, no history of heel trauma, no dislocation of the ankle joint, and no heel pain in the healthy population.

The study was approved by the Ethics Committee of People's Hospital of Rizhao. Patients who participated in this research had complete clinical data. The signed informed consents were obtained from the patients or the guardians.

Inspection method. DR used the DR-VR/S 2.0 digital X-ray camera produced by Philips, the Netherlands. The lateral view of the ankle joint or the lateral view of the heel was X-ray plain film, the focal length was $110 \mathrm{~cm}$, and the center line was at the talocalcaneal joint. Under the control of the automatic exposure system, the tube voltage varied from 45 to $60 \mathrm{KV}$, and the tube current varied from 1.5 to $4 \mathrm{mAs}$.

MRI adopted GE 1.5T magnetic resonance scanner and barrel coil, sagittal FSE T1WI (TR/TE=540 ms/9.9 ms), sagittal, coronal FSE T2WI (TR/TE=2,180 ms/27.8 ms), 
Table I. Sex distribution of the two groups from 8 to 15 years (number of cases).

\begin{tabular}{|c|c|c|c|c|c|c|c|c|c|}
\hline \multirow[b]{2}{*}{ Variables } & \multicolumn{9}{|c|}{ Age (years) } \\
\hline & $8 \sim$ & $9 \sim$ & $10 \sim$ & $11 \sim$ & $12 \sim$ & $13 \sim$ & $14 \sim$ & $15 \sim$ & Total \\
\hline \multicolumn{10}{|l|}{ Sever's } \\
\hline Male & 7 & 9 & 10 & 13 & 12 & 12 & 10 & 5 & 78 \\
\hline Female & 2 & 5 & 3 & 2 & 3 & 3 & 2 & 0 & 20 \\
\hline \multicolumn{10}{|l|}{ Control } \\
\hline Male & 7 & 9 & 9 & 11 & 11 & 10 & 10 & 6 & 73 \\
\hline Female & 5 & 6 & 6 & 7 & 8 & 5 & 6 & 4 & 47 \\
\hline
\end{tabular}

Table II. The distribution and number of calcaneus signs in Sever's disease group and control group between 8 and 15 years (number of cases).

\begin{tabular}{|c|c|c|c|c|c|c|c|c|c|}
\hline \multirow[b]{2}{*}{ Variables } & \multicolumn{9}{|c|}{ Age (years) } \\
\hline & $8 \sim$ & $9 \sim$ & $10 \sim$ & $11 \sim$ & $12 \sim$ & $13 \sim$ & $14 \sim$ & $15 \sim$ & Total \\
\hline \multicolumn{10}{|c|}{ Compact type } \\
\hline \multicolumn{10}{|l|}{ Sever's } \\
\hline Male & 7 & 8 & 10 & 13 & 12 & 12 & 10 & 5 & 77 \\
\hline Female & 2 & 4 & 3 & 2 & 3 & 3 & 2 & 0 & 19 \\
\hline \multicolumn{10}{|l|}{ Control } \\
\hline Male & 4 & 5 & 6 & 8 & 7 & 9 & 8 & 2 & 50 \\
\hline Female & 3 & 3 & 4 & 5 & 4 & 2 & 3 & 0 & 26 \\
\hline \multicolumn{10}{|c|}{ Cancellous type } \\
\hline \multicolumn{10}{|l|}{ Sever's } \\
\hline Male & 0 & 1 & 0 & 0 & 0 & 0 & 0 & 0 & 1 \\
\hline Female & 0 & 0 & 0 & 1 & 0 & 0 & 0 & 0 & 1 \\
\hline \multicolumn{10}{|l|}{ Control } \\
\hline Male & 3 & 4 & 3 & 3 & 4 & 1 & 2 & 1 & 23 \\
\hline Female & 2 & 3 & 2 & 2 & 3 & 3 & 3 & 3 & 21 \\
\hline \multicolumn{10}{|c|}{ Translucent line } \\
\hline \multicolumn{10}{|l|}{ Sever's } \\
\hline Male & 7 & 9 & 10 & 13 & 12 & 12 & 10 & 5 & 78 \\
\hline Female & 2 & 5 & 3 & 2 & 3 & 3 & 2 & 0 & 20 \\
\hline \multicolumn{10}{|l|}{ Control } \\
\hline Male & 1 & 3 & 4 & 3 & 3 & 1 & 0 & 0 & 15 \\
\hline Female & 1 & 2 & 3 & 3 & 1 & 0 & 0 & 0 & 10 \\
\hline
\end{tabular}

sagittal FSE STIR T2WI = 1,960 ms/31 ms, layer thickness was $4 \mathrm{~mm}$, FOV was $16 \mathrm{~cm}$.

Observation indicators. The manifestation of the Sever's disease and normal calcaneal epiphysis on the lateral X-ray film was observed: The density of the epiphysis (compact and cancellous bone type), and the radiolucent line of the epiphysis (Figs. 1-3) was observed. The characteristics of changes in MRI signal of Sever's disease were observed.

Statistical analysis. The statistical software package of SPSS 19.00 (IBM Corp., Armonk, NY, USA) was used. The $\chi^{2}$ test was used for the analysis between groups. $\mathrm{P}<0.05$ for the difference was statistically significant.

\section{Results}

Distribution of sex in Sever's disease group and control group at different ages (Table I). Among Sever's patients, the male incidence rate was $79.59 \%$, the female incidence rate was $20.41 \%$, the average age of onset was 11.35 , the average age of onset for male was 11.49, and the average age of onset for female was 10.80 .

The distribution and number of calcaneus epiphysis signs in Sever's disease group and control group at different ages (Table II).

Comparison of the density of calcaneus. In the Sever's group, the calcaneus epiphyses compact type: 77 males (78.57\%) and 


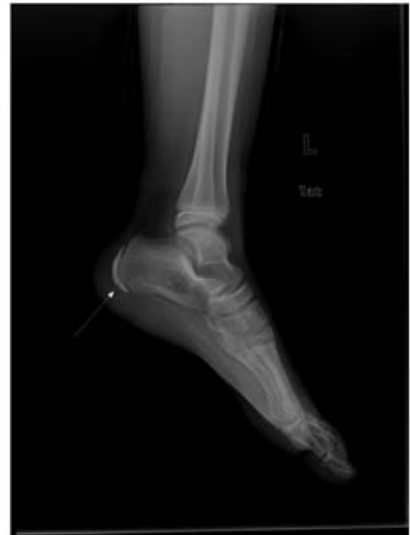

Figure 1. Male, 10 years old. Clinically diagnosed as Sever's disease, compact type of calcaneus epiphysis, see translucent line (arrow).

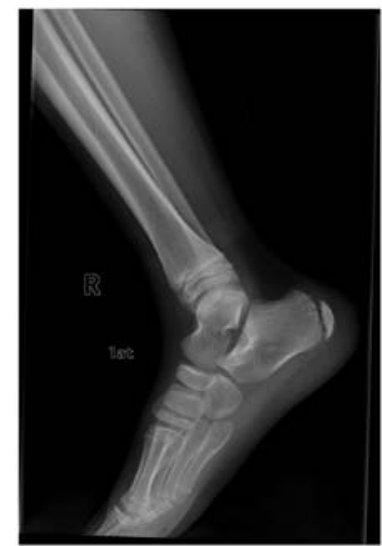

Figure 2. Female, 5 years old. Normal calcaneus epiphysis, compact type.

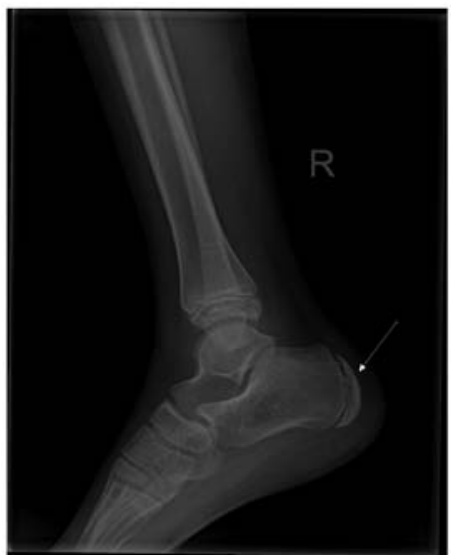

Figure 3. Female, 9 years old. Normal calcaneal epiphysis, cancellous bone type, see translucent line (arrow).

19 females (19.39\%); cancellous bone type: 1 male (1.02\%) and 1 female $(1.02 \%)$.

In the control group, the calcaneus epiphyses compact type: 50 males $(41.67 \%)$ and 26 females (21.67\%); cancellous bone type: 23 males (19.17\%) and 21 females (17.50\%) (Table II).

There was a statistically significant difference in the density of calcaneal epiphysis between the Sever's disease group and the control group $\left(\chi^{2}=38.85, \mathrm{P}<0.05\right)$.

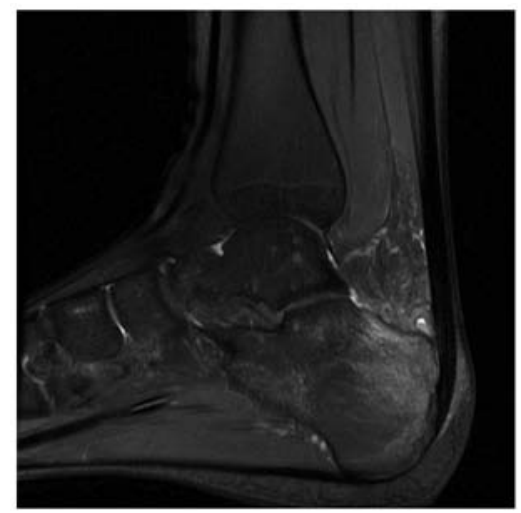

Figure 4. Male, 12 years old. Sag T1 FSE, normal calcaneus under MRI examination.

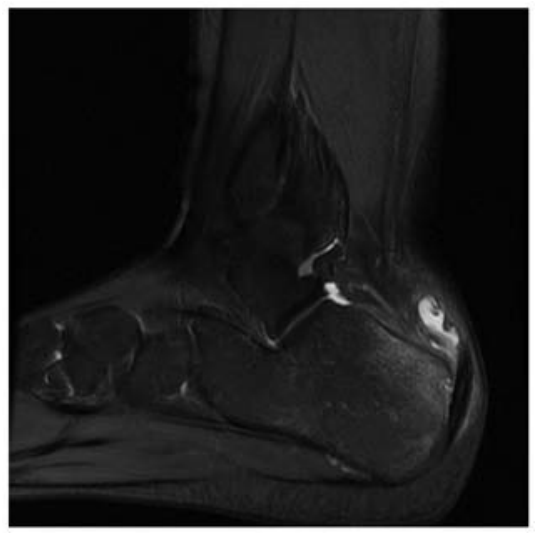

Figure 5. Male, 15 years old. Sag T2 STIR FSE, calcaneus bone marrow edema, edema of the lower Achilles tendon, effusion of the joint and posterior Achilles tendon bursa, soft tissue edema.

Comparison of the radiolucent line of the calcaneus. In the Sever's group, the translucent line of the calcaneus epiphysis was observed in: 78 males (79.59\%) and 20 females (20.41\%).

In the control group, the translucent line of the calcaneus epiphysis was observed in: 15 males (12.50\%) and 10 females (8.33\%).

There was a statistically significant difference between the Sever's disease group and the control group in terms of the radiolucent line $\left(\chi^{2}=137.51, \mathrm{P}<0.05\right)$.

Sever's disease signal changes on MRI images. The 6 patients who underwent MRI had different degrees of bone marrow edema, joint effusion and surrounding soft tissue swelling in MRI. Among them, 2 cases had high signal T2 weighed fat suppressed in the Achilles tendon attachment, and 1 case showed posterior Achilles tendon bursa effusion (Figs. 4 and 5).

\section{Discussion}

The results of this study suggested that the X-ray of Sever's disease showed an increase in the density of the epiphysis, a radiolucent line and a statistically significant comparison with the control group, which is consistent with previous 
studies (9,11-13). However, in the calcaneus epiphysis development of healthy children and adolescents, increased density and radiolucent line is also observed (4,7-9). It has been suggested in the literature that some patients with severe Sever's disease have reduced density and atrophy of the calcaneus epiphysis $(4,14)$. In the present study, patients with thinning and atrophy of the epiphysis have not been observed, which may be related to the small number of samples and the increased attention of parents. Radiolucent lines can also be seen in the developmental period of normal developing children's calcaneus, accounting for about $1 / 3$, usually 1 to 2 lines, and radiolucent lines were observed in all Sever's patients (11), with higher incidence in male than female, higher incidence in those with larger amount of exercise, obesity and in the preferential foot. Sever's disease is mostly bilateral, and the incidence of boys is higher than that of girls (15). The average age of onset of girls in this study was at the age of 10.08 years, and the average age of onset of boys was 11.49 years, which is consistent with the pattern of growth and development of girls earlier than boys.

Due to the high cost of MRI examination and the long waiting time required, there are few patients who choose this examination, and it is impossible to do statistical analysis. The signs and symptoms can only be observed according to a few cases. The nature of Sever's disease is the process of microfracture of bone cells and repairing after ischemic death. MRI has a good soft tissue resolution and is sensitive to bone marrow edema, synovial thickening, joint effusion and soft tissue edema (16). Sever's disease is a self-limiting disease, mainly characterized by pain, swelling, and fewer other symptoms such as fever. It is usually treated conservatively by appropriately limiting physical activity and suggesting rest to relieve the stimulation to the heel from pulling the Achilles tendon, or by physical methods such as scaffold support, cold compress, traction to alleviate the heel pain to achieve self-healing, most patients only need the clinician physical examination and the addition of lateral calcaneal view films, CT or MRI may be required for very few serious cases with poor prognosis from conservative treatment. Given the radioactivity of X-rays, it is still controversial whether Sever's patients need to take a film, but taking X-rays can rule out other lesions of the calcaneus such as fractures, osteomyelitis, calcaneal tumors or tumor-like lesions.

In conclusion, the main clinical manifestation of Sever's disease is local pain of the calcaneus. The X-ray is mainly characterized by increased density of the calcaneus epiphysis, and the appearance of radiolucent line. It is sometimes difficult to diagnose by X-ray film alone, the combination with the medical history or MRI examination is required. MRI is the most accurate imaging examination method for the damage of the epiphysis (17), which can provide more reliable image information for the observation and prognosis of Sever's disease osteophyte soft tissue injury, and provide direction for guiding clinical treatment $(18,19)$. This study was aimed to provide some help for clinical and imaging diagnosis of Sever's disease.

\section{Acknowledgements}

Not applicable.

\section{Funding}

No funding was received.

\section{Availability of data and materials}

The datasets used and/or analyzed during the present study are available from the corresponding author on reasonable request.

\section{Authors' contributions}

YG conceived the study and drafted the manuscript. JL and YL acquired the data. QL and SX analyzed the data and revised the manuscript. All authors read and approved the final manuscript.

\section{Ethics approval and consent to participate}

The study was approved by the Ethics Committee of People's Hospital of Rizhao (Rizhao, China). Patients who participated in this research had complete clinical data. The signed informed consents were obtained from the patients or the guardians.

\section{Patient consent for publication}

Not applicable.

\section{Competing interests}

The authors declare that they have no competing interests.

\section{References}

1. Ogden JA, Ganey TM, Hill JD and Jaakkola JI: Sever's injury: A stress fracture of the immature calcaneal metaphysis. J Pediatr Orthop 24: 488-492, 2004.

2. Mustapić M, Borić I, Lepur D, Zadravec D and Visković K: Sever's disease complicated with osteomyelitis. Acta Clin Croat 53: 252-255, 2014.

3. James AM, Williams CM and Haines TP: Heel raises versus prefabricated orthoses in the treatment of posterior heel pain associated with calcaneal apophysitis (Sever's Disease): A randomised control trial. J Foot Ankle Res 3: 3, 2010.

4. Cassas KJ and Cassettari-Wayhs A: Childhood and adolescent sports-related overuse injuries. Am Fam Physician 73: 1014-1022, 2006.

5. Kose O, Celiktas M, Yigit S and Kisin B: Can we make a diagnosis with radiographic examination alone in calcaneal apophysitis (Sever's disease)? J Pediatr Orthop B 19: 396-398, 2010.

6. Puffinbarger WR, Gruel CR, Herndon WA and Sullivan JA: Osteomyelitis of the calcaneus in children. J Pediatr Orthop 16: 224-230, 1996.

7. Ramponi DR and Baker C: Sever's Disease (Calcaneal Apophysitis). Adv Emerg Nurs J 41: 10-14, 2019.

8. Howard R: Diagnosing and treating Sever's disease in children. Emerg Nurse 22: 28-30, 2014.

9. Ceylan HH and Caypinar B: Incidence of calcaneal apophysitis in Northwest Istanbul. BMC Musculoskelet Disord 19: 267-272, 2018.

10. Perhamre S, Lazowska D, Papageorgiou S, Lundin F, Klässbo M and Norlin R: Sever's injury: A clinical diagnosis. J Am Podiatr Med Assoc 103: 361-368, 2013.

11. Liberson A, Lieberson S, Mendes DG, Shajrawi I, Ben Haim Y and Boss JH: Remodeling of the calcaneus apophysis in the growing child. J Pediatr Orthop B 4: 74-79, 1995.

12. Scharfbillig RW, Jones S and Scutter SD: Sever's disease: What does the literature really tell us? J Am Podiatr Med Assoc 98: 212-223, 2008. 
13. Weiner DS, Morscher M and Dicintio MS: Calcaneal apophysitis: Simple diagnosis, simpler treatment. J Fam Pract 56: 352-355, 2007.

14. Wiegerinck JI, Yntema C, Brouwer HJ and Struijs PA: Incidence of calcaneal apophysitis in the general population. Eur J Pediatr 173: 677-679, 2014.

15. Davison MJ, David-West SK and Duncan R: Careful assessment the key to diagnosing adolescent heel pain. Practitioner 260 30-33, 2016.

16. Pouletaut P, Claude I, Winzenrieth R, Ho Ba Tho MC and Sebag G: Automated analysis of MR image of hip: Geometrical evaluation of the Legg-Calve-Perthes disease. Med Eng Phys 27: 415-424, 2005

17. Park YH, Lim JW, Choi GW and Kim HJ: Quantitative magnetic resonance imaging analysis of the common site of Acute Achilles tendon rupture: 5 to $8 \mathrm{~cm}$ above the distal end of the calcaneal insertion. Am J Sports Med 47: 2374-2379, 2019.
18. Kose O: Do we really need radiographic assessment for the diagnosis of non-specific heel pain (calcaneal apophysitis) in children? Skeletal Radiol 39: 359-361, 2010.

19. Rossi I, Rosenberg Z and Zember J: Normal skeletal development and imaging pitfalls of the calcaneal apophysis: MRI features. Skeletal Radiol 45: 483-493, 2016.

(i) $\Theta($ This work is licensed under a Creative Common Attribution-NonCommercial-NoDerivatives 4.0 International (CC BY-NC-ND 4.0) License. 